\title{
A New Irrigation System (Endosplash) for a Rigid Endoscope in Trans-sphenoidal Endoscopic Surgery
}

\author{
Rintarou KuRODA, ${ }^{1}$ Takeshi NAKAJIMA, ${ }^{1}$ Takashi YAMAGUCHI, ${ }^{1}$ and Eiju WATANABE ${ }^{1}$ \\ ${ }^{1}$ Department of Neurosurgery, Jichi Medical University, Shimotsuke, Tochigi
}

\begin{abstract}
Obstruction of the visual field by blood is a major hindrance during endonasal endoscopic surgery, and a rapid and effective method for cleaning the lens is needed. We developed a new lens-cleaning system that does not employ a sheath or an irrigation-suction system. It is a 20-mm long cylinder with side holes that is attached to the barrel of the endoscope and is connected to a syringe containing saline. When the syringe is pressed, saline flows down to the tip along the barrel and washes the lens without requiring a sheath. We report the use of the system in six cases of endonasal endoscopic surgery. The lens was wiped significantly less often than during similar surgery performed without the use of this system. The Endosplash is simple and enables the surgeon to clean the lens with a single press of a syringe, thereby greatly enhancing the efficacy of endoscopic surgery.
\end{abstract}

Key words: endonasal endoscopic surgery, lens cleaning, endoscope irrigation system

\section{Introduction}

The development of new devices in recent years has led to a greater number of endonasal transsphenoidal pituitary surgeries being performed with an endoscope. ${ }^{1-3)}$ This method is less invasive and offers a wide field of view. ${ }^{4)}$

However, when an endoscope passes through the narrow corridor in the nasal cavity, the field of view is often blocked by blood adhering to the lens or conveyed along the barrel.

Obstruction of the visual field by dripping blood is an unavoidable byproduct of endonasal surgery, in which the tip of the endoscope usually faces downward. A lens obscured in this manner is usually cleaned by temporarily removing the endoscope from the nasal cavity and having an assistant wipe the lens. However, removing and reinserting the endoscope can be bothersome, and frequent removals and reinsertions can cause considerable psychological stress to surgeons and contribute to a longer operation. Such obscuring of a lens by blood is a major hurdle to beginners striving to master surgical techniques. A solution to this issue has been sought for many years.

The tip of an endoscope can be cleaned by attaching a sheath, ${ }^{5,6)}$ but using a sheath increases the outer

Received August 8, 2015; Accepted February 27, 2016 diameter of the endoscope, further narrowing the space available for surgery inside the already narrow nasal cavity. We developed a system, which we called Endosplash, which cleans the lens at the tip of the endoscope and creates a clear field of view without using a sheath.

\section{Materials and Methods}

The EndoArm ${ }^{\circledast}$ (Olympus Corp., Tokyo) was used for trans-sphenoidal pituitary surgery. This is a self-holding rigid endoscope with a $4-\mathrm{mm}$ barrel diameter and lenses with $0^{\circ}, 30^{\circ}$, and $70^{\circ}$ fields of vision. The present study was approved by the ethics committee of Jichi Medical University.

\section{Device}

The device was designed and tested mainly for endonasal surgery, in which the endoscope is inserted and held in the upper side of the nasal cavity. The device can also be used with the endoscope touching the lower wall of the nasal cavity.

The Endosplash was designed and manufactured by our team. The Endosplash is a cylindrical fitting that is $20 \mathrm{~mm}$ long and $10 \mathrm{~mm}$ in diameter which is attached to the proximal side of the endoscope (Fig. 1). We manufactured the Endosplash on Up Plus ${ }^{\circledR}$ 3D-printer (TierTime Technology Co., Ltd., Beijing, China) by using acrylonitrile-butadiene-styrene resin. 


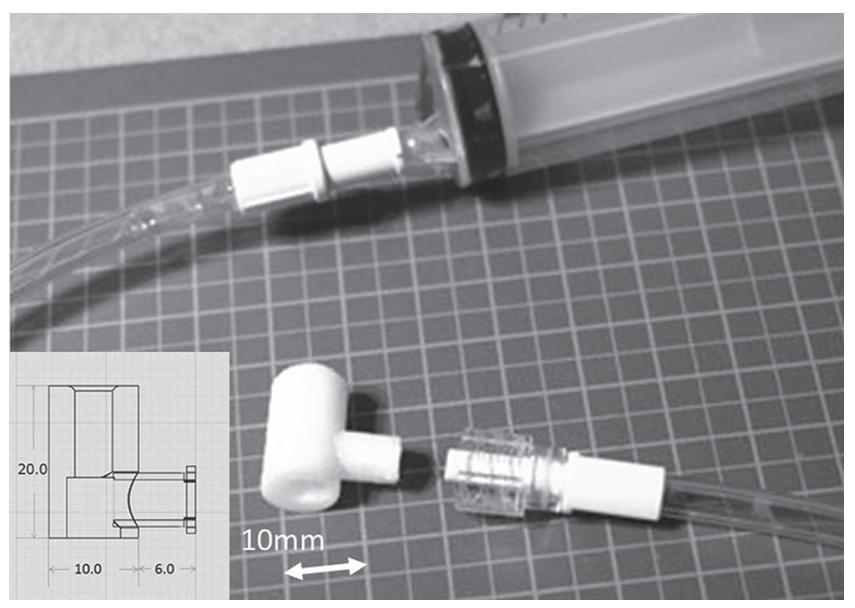

Fig. 1 Photograph of the Endosplash (inset showing internal structure), with method of attachment to the syringe.

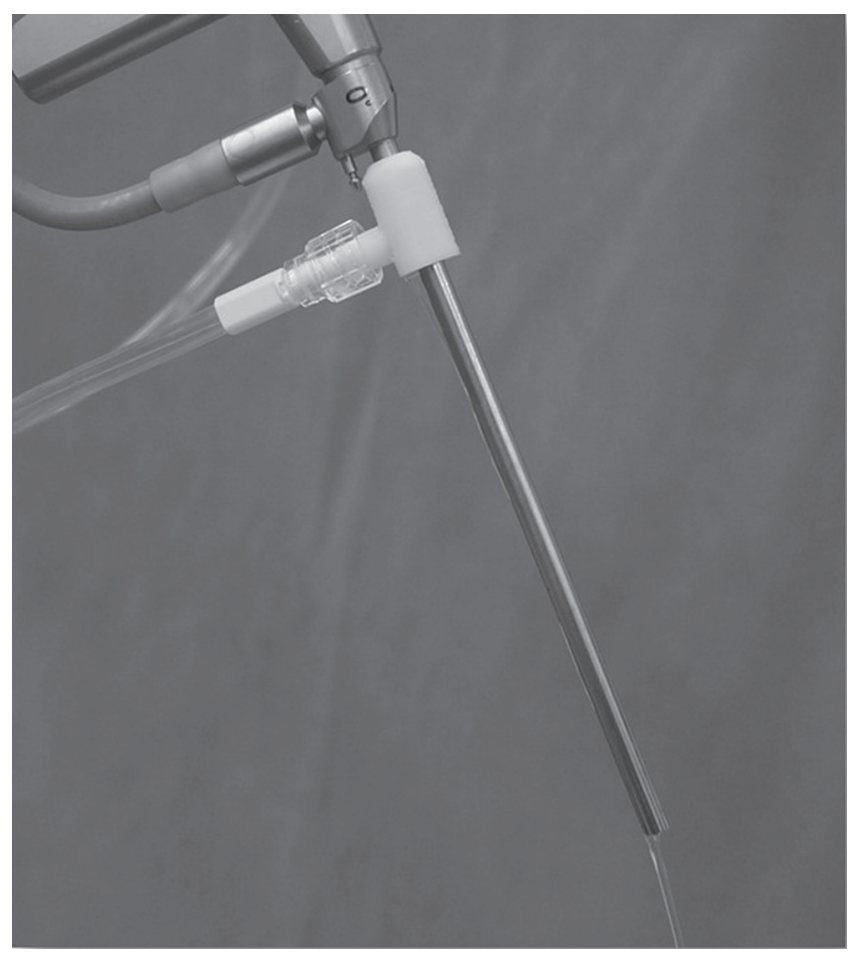

Fig. 2 Water stream along the barrel of the endoscope.

It has a side hole to which a commercially available drip infusion tube can be attached. The Endosplash whose shape is cylindrical and internal diameter is $4 \mathrm{~mm}$ can be securely attached to the endoscope (Fig. 2). Inside the cylinder is an L-shaped water channel about $2 \mathrm{~mm}$ in diameter that comes into vertical contact with the endoscope from the side hole and bends vertically to open in the direction of the tip of the endoscope. A water outlet is opened on the other side of the side hole. A saline-containing

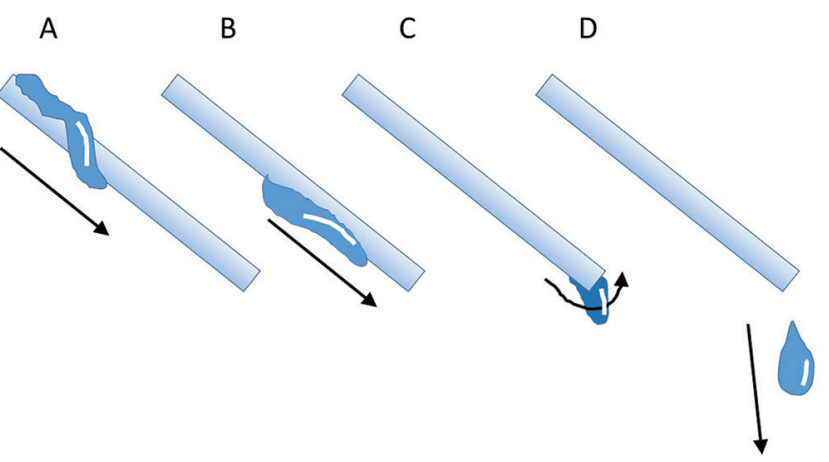

Fig. 3 Path of water stream. A: First along the upper surface, B: then along the lower surface, C: then creating a drop at the tip, and finally D: dripping down.

syringe $(20-30 \mathrm{ml})$ is attached to the extension tube, which causes the saline to flow. As a result, the water flows toward the tip of the endoscope along the lens barrel. It should be noted that the water stream does not drip down but travels along the barrel before eventually reaching the tip. Once there, the saline washes the surface of the lens and drips down (Fig. 3). This phenomenon occurs through the effect of surface tension and makes it possible to clean the lens easily, removing any blood that may have smeared the surface of the lens.

We used the Endosplash during endoscopic transnasal surgery, in which the endoscope is most often inserted through the upper part of the nostril. In this situation, because the saline travels along the lower surface of the barrel, the flow is not disrupted even if the upper surface of the barrel touches a wall of the nasal cavity (Fig. 4).

\section{Verification}

1. The optimum speed of water flow was examined quantitatively using a Codman ${ }^{\circledast}$ Malis ${ }^{\circledR} \mathrm{CMC}^{\circledast} \mathrm{V}$ Module $1000^{\mathrm{TM}}$ Irrigator (Malis irrigator; Codman \& Shurtleff, Inc., Raynham, Massachusetts, USA), which was connected to the side hole of the Endosplash. We measured the ability of flow speeds of $0.1-1$ $\mathrm{ml} / \mathrm{sec}$ to clean $0^{\circ}, 30^{\circ}$, and $70^{\circ}$ lenses.

2. We measured the optimal angle of the barrel from horizontal that would effectively clean $0^{\circ}, 30^{\circ}$, and $70^{\circ}$ lenses using the Malis ${ }^{\circledR}$ irrigator.

3. Using a test tube similar in shape to the nasal cavity, we examined how drops of water at the tip of the endoscope were removed by a suction tube to determine where the best suction effect was obtained.

4. Video records were retrospectively examined to clarify the effectiveness of the Endosplash. The frequency at which the endoscope was wiped was measured in three endonasal trans-sphenoidal 


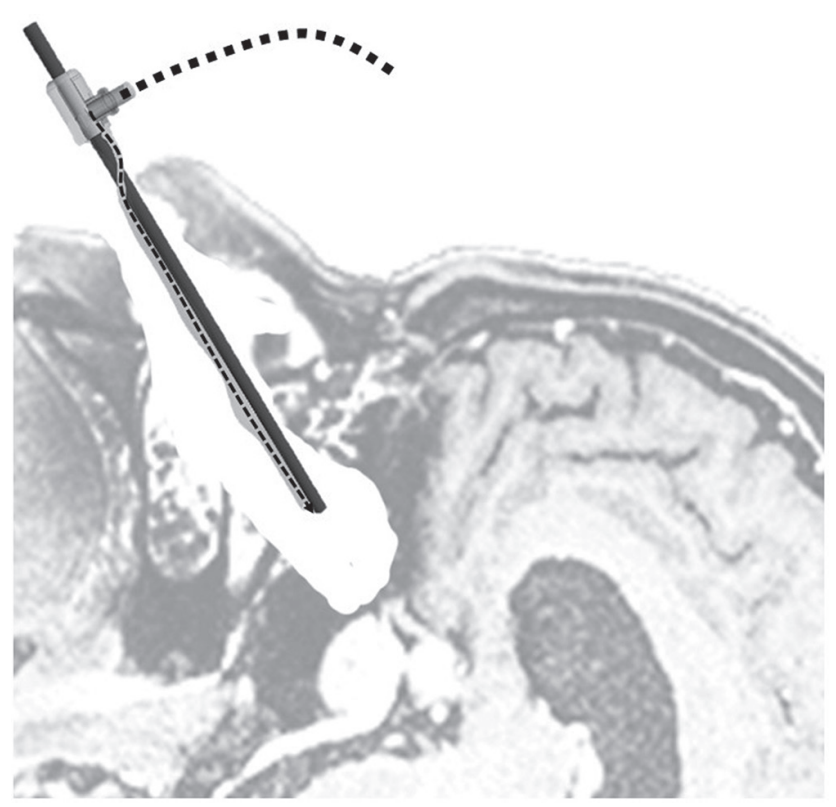

Fig. 4 Technique in case of being inserted from the upper part of the nostril. Because the water stream travels along the lower surface of the barrel, it not disrupted during its course.

surgeries using the Endosplash. We then selected the three consecutive cases prior to the introduction of the Endosplash, counted the number of times the endoscope was wiped during those procedures, and compared the results.

\section{Results}

\section{Endoscopic endonasal trans-sphenoidal pitui- tary surgery}

Mean number of wipes per hour in endoscopic endonasal trans-sphenoidal pituitary surgery was compared between surgeries performed with and without the Endosplash (Fig. 5).

Mean number of wipes per hour was significantly greater for the three cases in which the Endosplash was not used (11.7, 14, and 19 times per hour) compared with the Endosplash-assisted surgeries (2.9, 3.9 , and 3.3 times per hour; $\mathrm{p}=0.016$ ). However, in the three cases, the number of times that cleansing occurred in each case was 18.5, 6.7, and 13.0 times per hour, respectively. The number of times that the tip of the endoscope became dirty, measured in terms of the frequency of wiping combined with the frequency of cleansing, in the three cases with Endosplash was 21.4, 10.6 and 12.0 times per hour, respectively. The number of "wipe" plus "wash" with using Endosplash and the number of "wipe" without using Endosplash thus being more or less the same.

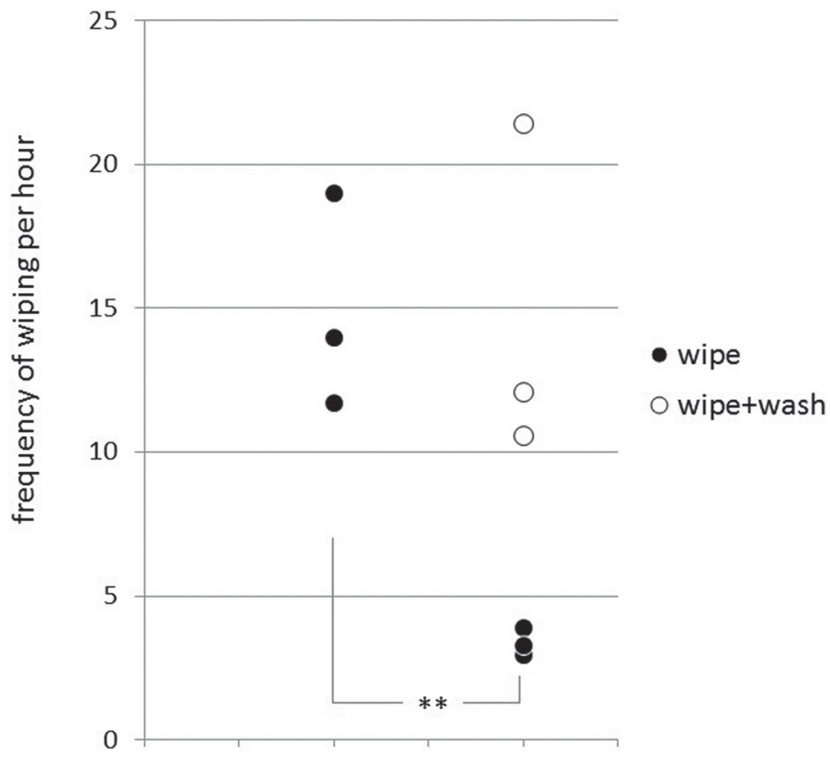

without with

Fig. 5 Graph showing instances of wiping the lens during three surgeries without the Endosplash and three surgeries with the Endosplash. Closed circles represent the number of wipes. Open circles represent the number of wipes plus washes.

\section{Dry-lab results}

1. Angle of the lens barrel

When endoscope barrel angle approaches the more horizontal, the more water drips down before it reaches the tip. When the angle was increased from horizontal to $30^{\circ}$ or more, it was found that the saline easily reached the tip of the endoscope. However, we discovered that water was able to reach the tip, even at angles of $<30^{\circ}$, with an increase in water flow.

\section{Optimal saline flow speed}

Using the Malis irrigator, sufficient cleaning of a $0^{\circ}$ lens required a minimum flow speed of $0.5 \mathrm{ml} / \mathrm{sec}$ when the angle of the endoscope barrel was $30^{\circ}$ from horizontal. The more vertical the barrel, the slower the water flow necessary for adequate lens cleaning. When the barrel angle was $60^{\circ}$ from horizontal, the minimum flow speed required for adequate lens cleaning was $0.1 \mathrm{ml} / \mathrm{sec}$. There is no particular upper limit to flow speed, but no major changes in cleaning effect were noted when the flow speed was increased any further. Results were nearly the same for a $30^{\circ}$ endoscope lens. For a lens with a $70^{\circ}$ field of view, the barrel angle had to be $>70^{\circ}$ from horizontal, with some exceptions. 


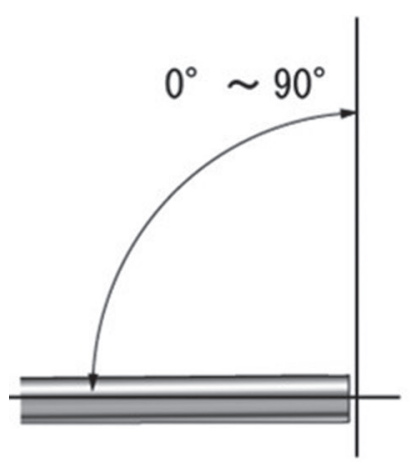

A

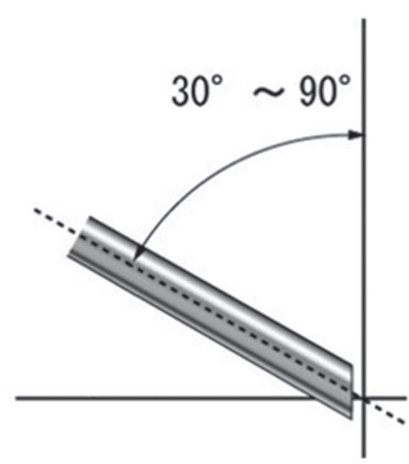

B

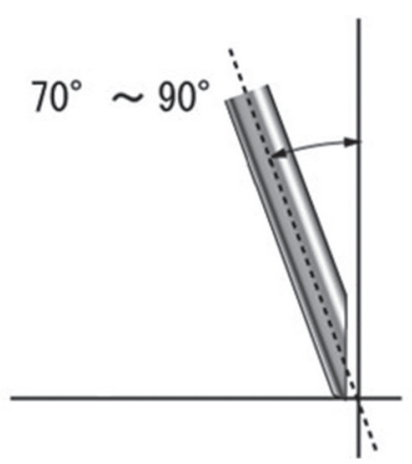

C

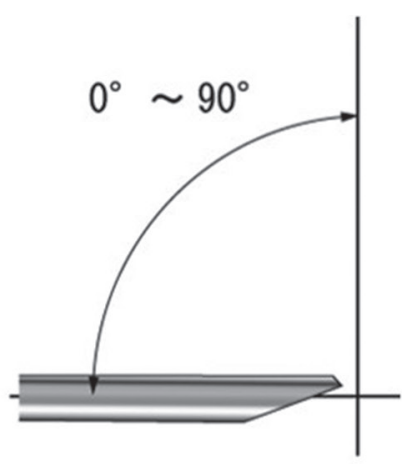

D

Fig. 6 Showing the range of angles possible to wash $0^{\circ}, 30^{\circ}$, and $70^{\circ}$ endoscope lenses. The angle ranges are $0^{\circ}-90^{\circ}, 30^{\circ}-90^{\circ}$, and $70^{\circ}-90^{\circ}$ for $0^{\circ}, 30^{\circ}$, and $70^{\circ}$ lenses, respectively (A, B, and C). Rotating the endoscope inside the nasal cavity so that the lens faces downward allows cleaning of a $70^{\circ}$ lens (D).

The length of time from start of release of the saline until the lens was cleaned and a clear visual field achieved was approximately $1 \mathrm{sec}$, regardless of the angle of the lens barrel from horizontal.

3. Cleaning based on the direction of view of the lens We examined this system for $0^{\circ}, 30^{\circ}$, and $70^{\circ}$ endoscopic lenses. Even if the saline reaches the tip of the endoscope, it is not possible to clean the surface of the lens unless the saline is conveyed up to the surface of the lens and drops are formed. This means that, depending on the direction of view of the lens, in some cases the surface of the lens cannot be cleaned even if the water reaches the tip. If the lens angle is $0^{\circ}$, cleansing of the lens is possible at all angles, from horizontal to $90^{\circ}$ (Fig. 6A). For a $30^{\circ}$ lens with the lens barrel less than $30^{\circ}$ from horizontal, the surface of the lens will be more than $90^{\circ}$ from the horizontal surface, meaning that drops of water will not form on the lens surface. Accordingly, cleaning of lens surfaces ranging from $30^{\circ}$ to $90^{\circ}$ from horizontal was possible (Fig. 6B). For a $70^{\circ}$ lens, cleaning was possible when the lens barrel of the endoscope was between $70^{\circ}$ and $90^{\circ}$ (Fig. 6C).

Thus, although an endoscope with a lens angle of $70^{\circ}$ off horizontal could not be cleaned at the angle employed when the endoscope was actually in use, cleaning was possible by provisionally rotating the lens barrels inside the nasal cavity so that the surface of the lens faced downward and then returning it to its former position (Fig. 6D).

\section{Removing drops of water at the tip}

Drops of water are initially formed at the tip, and the moment they drip down they clear the field of view (Fig. 3). However, until the drops of water are obtained, the field of view shakes as when one sees through tear-filled eyes. Without assistance, the drops of water will drip down naturally after 1-2 sec, but faster cleaning is possible if they are made to fall more rapidly. We wanted to determine how to remove the drops of water at the tip the most quickly. We confirmed that drops of water can be sucked away and removed instantaneously simply by passing a suction tube close to the tip of the endoscope in the narrow passage of the nostril. We experimented with a test tube of $20-\mathrm{mm}$ inner diameter and found that drops of water could be sucked away and removed if the distance between the tip of the endoscope and the suction tube was $15 \mathrm{~mm}$ or less.

\section{Representative case}

A 68-year-old male was referred to our clinic with hemianopsia. Magnetic resonance imaging showed a $15-\mathrm{mm}$ diameter pituitary tumor. Tumor removal was performed with an endonasal trans-sphenoidal surgery.

With the patient in supine position, the head was fixed with a MAYFIELD ${ }^{\circledR}$ Modified Skull Clamp. An endoscope was inserted into the right nasal cavity. The sphenoid sinus was opened through the natural opening and the sellar floor was fenestrated. After the dura mater had been opened, a soft, white tumor mass emerged. The tumor was completely removed with curettage. A normal pituitary gland was noted inside the sella.

The operation was performed with the Endosplash attached to the endoscope (Fig. 7). Clouding of the lens from contact with the nasal mucosa and dripping of blood occurred 54 times in $143 \mathrm{~min}$ 
and was resolved by cleaning with the Endosplash 47 times. However, there were seven instances in which strong attachment of hard clot required removal of the endoscope to wipe the lens.

\section{Discussion}

In endonasal endoscopic surgery, the lens often becomes smeared with blood, thus obstructing the

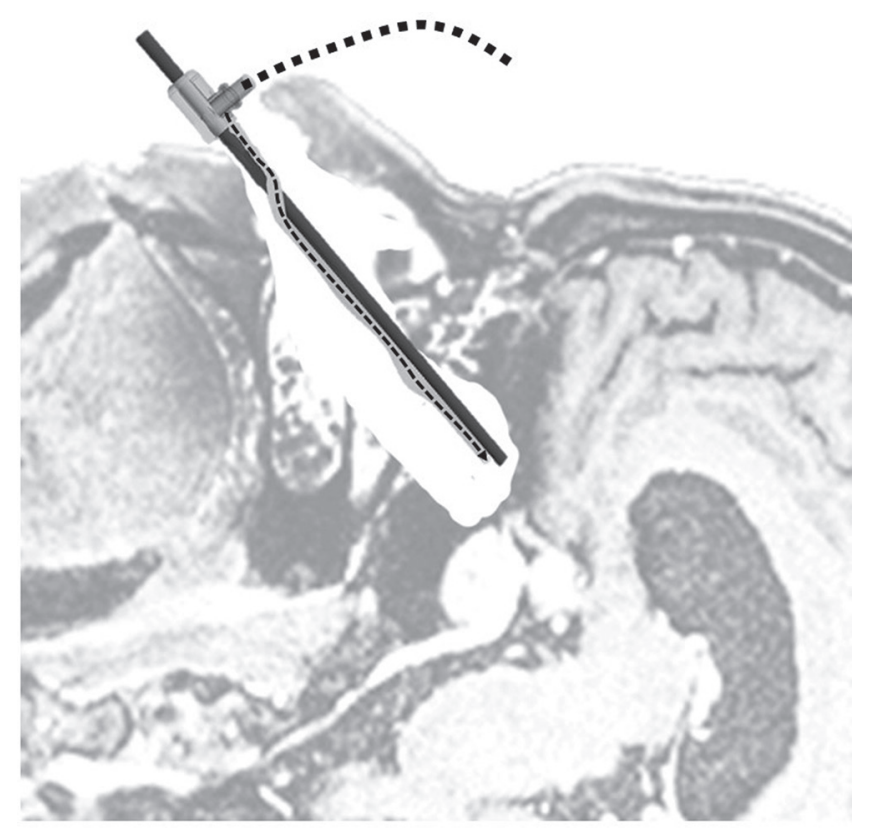

Fig. 7 Technique in case of being inserted from below the nostril. field of view and making it necessary to remove the endoscope and wipe the lens. The amount of time and effort involved in this procedure has been a major problem in the past.

The Endosplash was intended to improve surgical efficiency by reducing the operator's stress and to assist trainees in acquiring operative skills. While the efficiency of a surgical procedure is difficult to measure directly, the decreased frequency with which the endoscope had to be removed and reinserted, compared with procedures in which the Endosplash was not used, suggests that the Endosplash was associated with enhanced surgical efficiency.

One method that has attempted to solve this problem involves the use of a sheath that will cleanse the endoscope. Although this method enables efficient cleaning of the lens without having to remove the endoscope, its disadvantage is the increased outer diameter of the endoscope after attachment of the sheath. For example, the maker of K-Endosheath ${ }^{\circledR}$ (Koken Co., Ltd., Bunkyo-ku, Tokyo) having systems using a sheath, emphasize its thinness, but the outer diameter of the sheath is 1.5 times larger than the naked barrel of endoscope (Fig. 8). The thicker the outer diameter of the endoscope is, the more difficult the surgical procedure is. Considering the narrowness of the nasal cavity, increasing the outer diameter, thereby reducing operability inside the nasal cavity, is a major disadvantage.

By connecting an irrigation system to the Endosplash, an operator can perform the surgical procedure
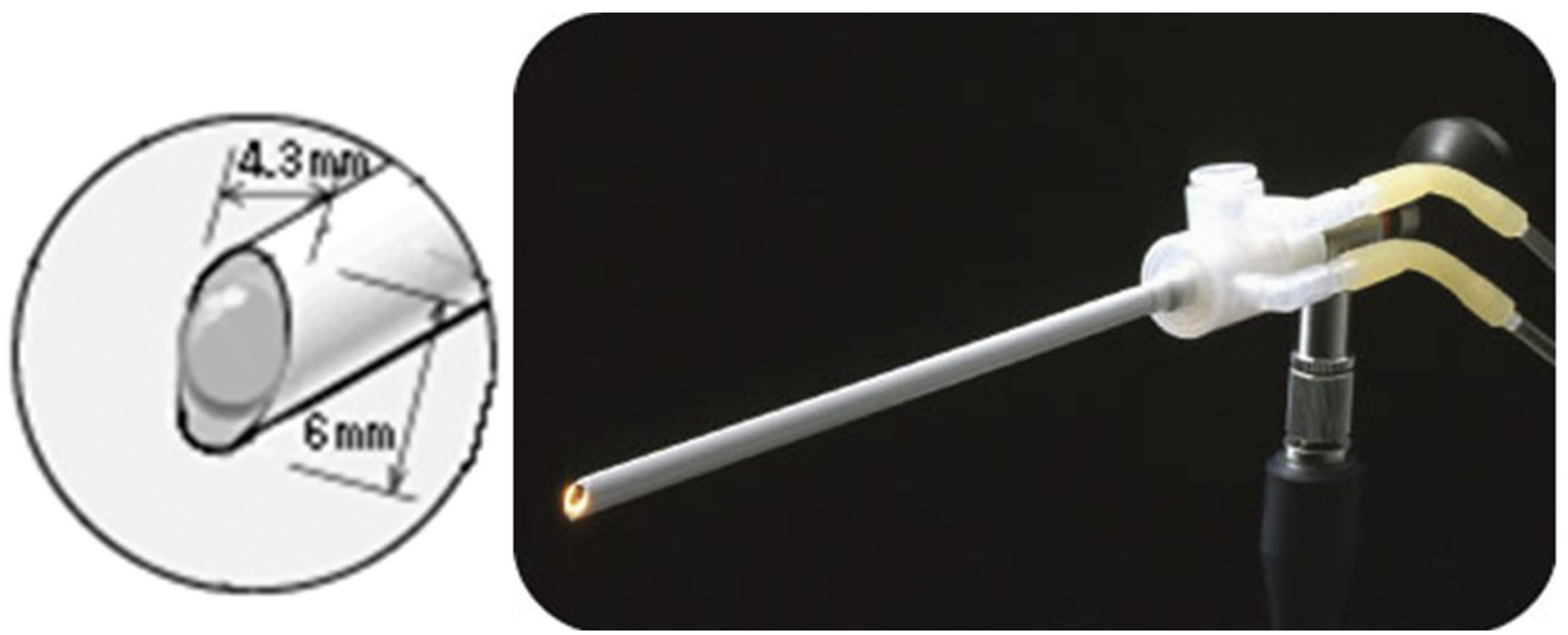

Fig. 8 Whole image and detail of the top of K-Endosheath ${ }^{\circledR}$ quoted and modified from the web site. (KOKEN K-Endosheath <http://www.kokenmpc.co.jp/english/products/medical_plastics/ent/k-endsheath/index.html>)(2016.1.25) 
by him- or herself, but this can be burdensome because another irrigation system is required. The Endosplash can be simply used by having an assistant press the syringe.

During surgery, we inadvertently dripped water onto the barrel of an endoscope and discovered that the tip of the endoscope had been cleaned. Employing this method, the saline not only reaches the tip of the endoscope, but also moves around the lens surface to clean the tip. This technique is made possible entirely through surface tension.

The endoscope points downward in most surgeries, meaning that the cleaning water travels along the lens barrel and flows to the tip of the endoscope. We considered whether the saline would flow to the tip of the endoscope if the lens barrel were horizontal. We found that if we slightly increased the speed of the water flow, the water would reach the tip, even with the barrel in a horizontal position, and also that it was easy for the saline to reach the tip if the angle was at least $30^{\circ}$ from horizontal.

In terms of the direction of the lens, it was necessary for the lens to be facing downward in order for the surface of the lens to be cleaned by means of surface tension. We found that the lens surface could be cleaned if the lens barrel was horizontal for a $0^{\circ}$ lens, if it was $\geq 30^{\circ}$ from horizontal for a $30^{\circ}$ lens and $\geq 70^{\circ}$ off horizontal for a $70^{\circ}$ lens. Under normal circumstances, the endoscope is angled at $45^{\circ}$ or more, which facilitates lens cleaning.

Water that has passed along the lens barrel goes around the surface of the lens and temporarily forms drops. The field of view is obstructed while the drops of water remain on the surface of the lens. However, our results indicate that a suction tube is very effective at rapidly removing water drops from the surface of the lens.

This may be caused by a strong air current arising inside a narrow space (Venturi effect), which increases the range accessible to negative pressure caused by the suction tube.

Because the saline reaches the tip of the endoscope after passing along its lower surface of the barrel, it is essential that the lower surface of the barrel not come into contact with any nasal surface. Endoscopes are normally used in such a way that they pass through the upper part of the nasal cavity. However, depending on the surgical technique being employed, it may pass through the lower part. If the lower surface of the barrel comes into contact with the nostril, it will not be possible to convey water to the tip. There are two possible solutions to this problem.

Method 1: When cleaning the lens, the endoscope can temporarily be moved slightly upward to create a space between the barrel and the nostril.

Method 2: The Endosplash should be set so that it comes to within $2 \mathrm{~cm}$ of the nostril during cleaning. The water outlet is set to the top surface of the lens barrel; water will initially flow along the top surface of the lens barrel (Fig. 7). After entering the nasal cavity it will pass along the lower surface of the lens barrel and thereby enable cleaning.

For a $70^{\circ}$ lens, our results suggest that cleansing is possible if the barrel angle is more than $70^{\circ}$ from horizontal, which is not practical (Fig. 6C). However rotating the endoscope inside the nasal cavity so that the lens faces downward (Fig. 6D) allows cleaning and can dramatically decrease the number of endoscope removals and reinsertions.

\section{Conclusion}

The major benefit of this system is that it makes it possible to clean the tip of an endoscope inside the nasal cavity without increasing the outer diameter of the endoscope itself, thereby dramatically reducing the number of removals and reinsertions of the endoscope. The Endosplash can enable experienced surgeons to increase their operational efficiency and reduce stress, and will free surgeons undergoing training from unnecessary stress and allow necessary skills to be acquired in an efficient manner.

\section{Conflicts of Interest Disclosure}

The authors have no personal, financial, or institutional interest in any of the drugs, materials, or devices described in this article.

\section{References}

1) Abe T, Matsumoto $K$, Shimazu M, Jimbo H, Sunaga S, Dohi K, Sasaki K, Izumiyama H, Ohki S, Fujitani S: [Transnasal microsurgery using a micro-pressuresuction-irrigation system for pituitary adenomas]. No Shinkei Geka 27: 225-231, 1999

2) Cappabianca P, Cavallo LM, Solari D, Stagno V, Esposito F, de Angelis M: Endoscopic endonasal surgery for pituitary adenomas. World Neurosurg 82: S3-S11, 2014

3) Kanter AS, Dumont AS, Asthagiri AR, Oskouian RJ, Jane JA Jr, Laws ER: The transsphenoidal approach. A historical perspective. Neurosurg Focus 18: e6, 2005 
4) Kubo S, Kikawada T, Hasegawa H, Tominaga S, Yoshimine T: Irrigation-suction straw sheath system for a rigid endoscope during endonasal endoscopic pituitary surgery. Minim Invasive Neurosurg 48: 373-375, 2005

5) Lanzino G, Laws ER: Pioneers in the development of transsphenoidal surgery: Theodor Kocher, Oskar Hirsch, and Norman Dott. J Neurosurg 95: 1097-1103, 2001
6) Liu JK, Das K, Weiss MH, Laws ER, Couldwell WT: The history and evolution of transsphenoidal surgery. J Neurosurg 95: 1083-1096, 2001

Address reprint requests to: Eiju Watanabe, Department of Neurosurgery, Jichi Medical University, 3311 Yakushiji, Shimotsuke, Tochigi 329-0498, Japan. e-mail: eijuwat@gmail.com 